\title{
Cosmic Ray helium spectrum measured by the DAMPE experiment
}

\author{
Margherita Di Santo, ${ }^{a, b}, *$ Peng-Xiong Ma, ${ }^{c}$ Antonio Surdo, ${ }^{d}$ Chuan Yue ${ }^{c}$ and Ya-Peng \\ Zhang ${ }^{e}$ on behalf of the DAMPE $^{\dagger}$ Collaboration \\ (a complete list of authors can be found at the end of the proceedings)
}

${ }^{a}$ Gran Sasso Science Institute (GSSI), Via Iacobucci 2, I-67100 L’Aquila, Italy

${ }^{b}$ Istituto Nazionale di Fisica Nucleare (INFN) -Laboratori Nazionali del Gran Sasso, I-67100 Assergi, L'Aquila, Italy

${ }^{c}$ Key Laboratory of Dark Matter and Space Astronomy, Purple Mountain Observatory, Chinese Academy of Sciences, Nanjing 210023, China

${ }^{d}$ Istituto Nazionale di Fisica Nucleare (INFN) - Sezione di Lecce, I-73100, Lecce, Italy

${ }^{e}$ Institute of Modern Physics, Chinese Academy of Sciences, Nanchang Road 509, Lanzhou 730000, China E-mail: margherita.disanto@gssi.it, mapx@pmo.ac.cn, antonio.surdo@le.infn.it, yuechuan@pmo.ac.cn, y.p.zhang@impcas.ac.cn

DAMPE (DArk Matter Particle Explorer) is a Space mission project promoted by the Chinese Academy of Sciences (CAS), in collaboration with Universities and Institutes from China, Italy and Switzerland. The satellite hosting the DAMPE detector has been successfully launched on December 17th, 2015, and is currently smoothly operating and collecting data. The main goals of the mission are: indirect search of Dark Matter, looking for signatures in the electron and photon spectra with energies up to $10 \mathrm{TeV}$; high energy gamma-ray astronomy; analysis of the flux and composition of primary Cosmic Rays (CR) in the energy range from a few tens of $\mathrm{GeV}$ up to hundreds of $\mathrm{TeV}$. In this work we present the latest result on the CR Helium spectrum measured by DAMPE and discuss the observed features in the light of the current models about the origin, acceleration and propagation of galactic Cosmic Rays. The outcome is validated through independent analyses performed within the DAMPE Collaboration, which give consistent results within the analysis uncertainties.

$3^{\text {th }}$ International Cosmic Ray Conference (ICRC 2021)

July 12th-23rd, 2021

Online - Berlin, Germany

\footnotetext{
*Presenter

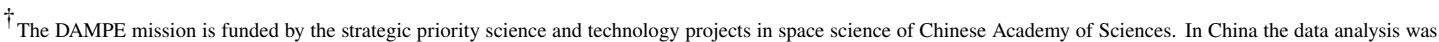
supported in part by the National Key Research and Development Program of China (No. 2016YFA0400200), the National Natural Science Foundation of China (Nos. 11525313, 11622327, 11722328, U1738133, U1738205, U1738207, U1738208), the strategic priority science and technology projects of Chinese Academy of Sciences (No. XDA15051100), the 100 Talents Program of Chinese Academy of Sciences, and the Young Elite Scientists Sponsorship Program. In Europe the activities and the data analysis are supported by the Swiss National Science Foundation (SNSF), Switzerland; the National Institute for Nuclear Physics (INFN), Italy.
} 


\section{Scientific framework}

Galactic Cosmic Rays (GCRs) travel through the Milky Way bringing key information about their sources and their accelerators. Measurements of their energy spectra already revealed special features that represent the best clues for a deeper learning of the properties of our Galaxy. These features consist in unexpected deviations from the single power-law behavior of the spectra predicted by theoretical models of shock accelerations of particles with energies below the so-called knee at energies of about $3-4 \mathrm{PeV}$. One of these features is the spectral hardening, pointed out at energies of hundreds of $\mathrm{GeV} /$ nucleon by precise measurements of the energy spectra of protons, helium and heavier nuclei provided by several experiments [1]-[5]. At TeV-energies, a further feature named spectral softening has been revealed in the proton energy spectrum and some hints of a similar feature were also observed in the helium spectrum, although with very high uncertainties [2][5][6]. This scenario strongly encourages measurements with higher precision at higher energies.

In this work, we present the measurement of the helium energy spectrum measured by the DAMPE experiment in the energy range from $70 \mathrm{GeV}$ to $80 \mathrm{TeV}$ [7].

\section{The DArk Matter Particle Explorer}

The DArk Matter Particle Explorer (DAMPE) [8] is a Space mission designed to investigate several scientific fields. Since December 2015, DAMPE allows the direct measurement of Cosmic Ray spectra up to energies of hundreds of TeV. The DAMPE payload consists of four sub-detectors, instrumental for the particle identification process. CRs entering the DAMPE satellite from its top firstly cross the Plastic Scintillator Detector (PSD) [9]. It consists of two layers of plastic scintillator bars arranged such that the bars in the first layer are orthogonal to those of the second plane. The PSD is the main responsible of charge identification by measuring the absolute value of $Z$, also providing an anti-coincidence veto signal for $\gamma$-rays. A second sub-detector named Silicon Tungsten tracKer-converter (STK) [10] provides the trajectory reconstruction of the crossing CR. It is made of 6 tracker planes and 3 tungsten layers, the latter chosen to enhance the photon conversion in electron-positron pairs. The energy deposited by the incoming particle is then measured by the core of the DAMPE experiment, a deep $B G O$ calorimeter [11] composed of 14 layers of $\mathrm{Bi}_{3} \mathrm{Ge}_{4} \mathrm{O}_{12}$ crystal bars. The last sub-detector is the NeUtron Detector (NUD) [12] made of boron-doped plastic scintillator tiles improving the discrimination power for hadronic and electromagnetic showers.

\section{Data sample}

In this work we analyzed 54 months of on-orbit data collected by the DAMPE satellite while orbiting around the Earth in the period January 1st, 2016 - June 30th, 2020. The satellite operations are affected by an instrumental dead time covering $3.0725 \mathrm{~s}$ for each event, corresponding to $\sim 17.2 \%$ of the total operational time (O.T.), while $\sim 1.7 \%$ of the O.T. is dedicated to on-orbit calibrations. Moreover, we exclude from our data sample all the events recorded by the detector while crossing the South Atlantic Anomaly (SAA) region ( 4.9\% O.T.) together with the data recorded during the period from September 9th, 2017 to September 13th, 2017 because of a giant solar flare affecting the operational status of the instrument. Hence, by taking into account all of these information, the resulting livetime corresponding to our data sample is $1.08 \times 10^{8} \mathrm{~s}$, namely $\sim 76 \%$ of the total O.T. 
The results obtained from the analysis of the described flight data sample have been supported by the analysis and comparison with Monte Carlo (MC) simulation data generated by using the GEANT4 toolkit [13] with the FTFP_BERT physics list for helium nuclei in the primary energy range from $10 \mathrm{GeV}$ to $500 \mathrm{TeV}$ and for proton events from $10 \mathrm{GeV}$ to $100 \mathrm{TeV}$ [5]. We also simulated proton events from $100 \mathrm{TeV}$ of incident energy up to $1 \mathrm{PeV}$ by implementing the DPMJET3 model via the CRMC-GEANT4 interface. Further simulations have been performed with FLUKA [14] in order to study the systematic uncertainty related to the hadronic model.

\section{Event selection}

\section{- Pre-selection}

The first step of the analysis consists in the pre-selection of well reconstructed events. On top of the preliminary requirement list there is the activation of the High Energy Trigger (HET), which occurs when the energy deposits in the first three BGO layers are greater than $\sim 13$ proton Minimum Ionizing Particles (MIPs) and higher than 2.4 MIPs in the fourth one, where $1 \mathrm{MIP}_{\mathrm{BGO}} \sim 23 \mathrm{MeV}$. Moreover, in order to avoid the geomagnetic cut-off effect [15], we exclude from the analysis all the events with an energy deposition inside the first 13 BGO layers lower than $20 \mathrm{GeV}$. We also ask for events with top-down developments by requiring that the total energy deposited inside the first two BGO layers is lower than the energy released in the third and fourth layers. Finally, in order to rule out events of particles entering the detector from the sides, we require that the energy deposition in each layer is less than $35 \%$ of the total energy deposited inside the first 13 layers of the calorimeter. It is important to point out that in this analysis we do not consider the last layer of the calorimeter for the energy measurements. This choice was made because of the high gain of this layer which might imply more critical issues related to saturation effects.

\section{- STK track selection}

The second step of the analysis is the STK track selection, consisting on several requirements guiding the choice of events with a well reconstructed track for which a good match is guaranteed with the signals provided by the PSD and the BGO. First of all, the events are required to have more than 2 hits for the reconstructed track provided by the STK tracking algorithm. If this requirement is satisfied by more than one track, we choose the one with the maximum total analog-to-digital converter and a reduced $\chi^{2}$ smaller than 35 . As previously mentioned, a good match of the candidate track with the axis of the shower developed inside the BGO is needed. For this reason we ask that the projections of the STK and BGO tracks on both the PSD layers are distant less than $90 \mathrm{~mm}$ and also that the centroids of the energy deposits in the first four BGO layers are located at a distance from the projected STK track lower than $25 \mathrm{~mm}$. Finally, we demand for a full containment of the track inside the PSD, the STK and the BGO in order to select events with a good containment of the shower inside the full detector.

\section{- Helium event selection}

At this stage of the analysis, we are ready to select helium candidates from the surviving event sample. For this purpose, we use the charge measurements provided by the PSD and the 

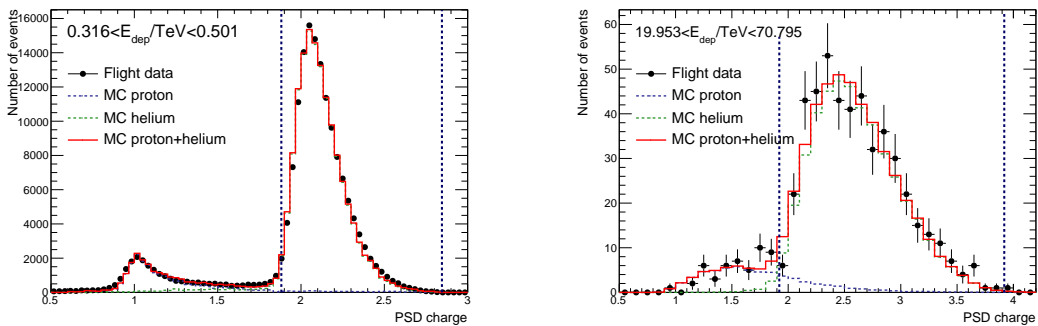

Figure 1: PSD charge distributions in two different BGO energy deposition ranges. The charge value is defined as the minimum of the charge measurements provided by the two PSD layers. Black points represent on-orbit data, compared with the best-fit proton MC (in blue), helium MC (in green) and proton+helium MC (in red). The range in which helium candidate events are selected is described by the vertical dashed blue lines representing the charge selection cut.

STK. More in detail, we firstly use the STK charge measurement by asking that the signal in ADC counts for the first hit of the STK track is greater than 120, which is important in order to dramatically suppress the background due to proton events in our sample. An energydependent PSD charge selection is then carried out by studying the energy loss distributions in both the PSD layers, proportional to the square of the particle charge according to the Bethe-Bloch formula, for several ranges of the BGO energy deposition. The charge selection cut is hence described by the following formula:

$$
1.85+0.2 \cdot \log \left(\frac{E_{B G O}}{10 \mathrm{GeV}}\right)<P S D_{\text {Charge }}<2.8+0.007\left[\log \left(\frac{E_{B G O}}{10 \mathrm{GeV}}\right)\right]^{4.0}
$$

where $P S D_{\text {Charge }}$ is the charge reconstructed in each PSD layer and it's not equivalent to the real charge of the incoming particle. The last requirement is that the reconstructed PSD charge for each layer has a value within a factor of 2. Fig.1 shows two examples of the PSD charge distributions in two different BGO energy bins for flight data and $\mathrm{MC}$ simulations of proton and helium events, where the energy-dependent charge selection cut of Eq. 1 is described by the vertical dashed lines.

\section{Proton background}

The final helium candidate event sample satisfying all the analysis selection criteria described up to now includes a fraction of mis-identified proton events representing the main contribution to the background. The pollution due to electrons and heavier nuclei is negligible. Fig.2 shows the residual proton contamination obtained by studying the PSD charge distributions and using the best-fit proton templates. The fraction of proton events contaminating our helium sample ranges between $\sim 0.05 \%$ for total BGO energy depositions of $20 \mathrm{GeV}$ up to $\sim 4 \%$ for $60 \mathrm{TeV}$.

\section{Efficiencies}

\subsection{High Energy Trigger efficiency}

The HET efficiency has been estimated by means of another trigger implemented in DAMPE, namely the Unbiased trigger (UNBT). The requirement for the activation of the UNBT is that the energy depositions inside the first two BGO layers are greater than $\sim 0.4 \mathrm{MIPs}(\sim 9.2 \mathrm{MeV})$ in each hit BGO bar, so this is the most inclusive trigger. The UNBT is pre-scaled by $1 / 512$ when the satellite is orbiting in the geographical latitude range $\left[-20^{\circ}, 20^{\circ}\right]$ and $1 / 2048$ at higher latitudes. 


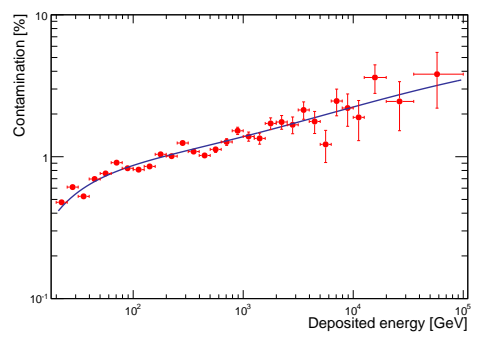

Figure 2: Red points represent the proton contamination as a function of the energy deposited inside the BGO calorimeter. The blue line is a forth degree polynomial fit function.
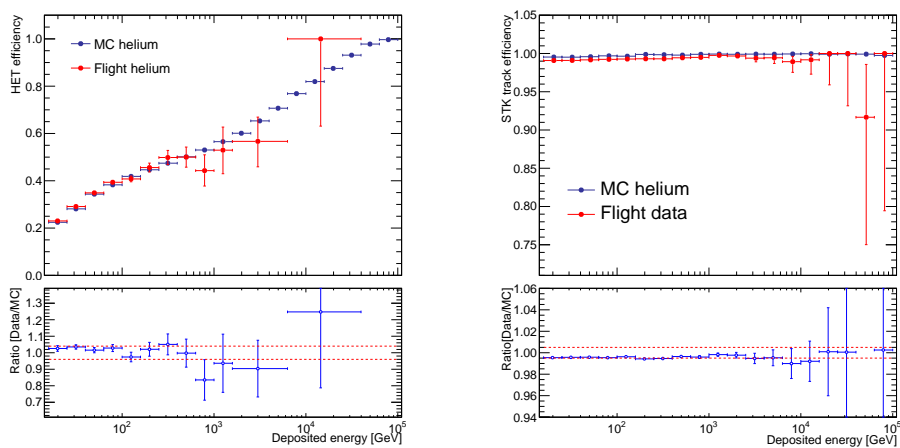

Figure 3: (Left) High Energy Trigger efficiency as a function of the BGO energy deposition for the selected helium candidate events. The deviation of on-orbit data (in red) from MC simulations (in blue) is found to be within $\sim 4 \%$ below $1 \mathrm{TeV}$. (Right) Track reconstruction efficiency as a function of the BGO energy deposition. The difference between on-orbit data (in red) and MC simulations (in blue) is found to be within $\sim 0.5 \%$.

We compute the HET efficiency in the following way:

$$
\varepsilon_{H E T}=\frac{\mathrm{N}_{\text {HET } \mid \text { Unb }}}{\mathrm{N}_{\text {Unb }}}
$$

where $\mathrm{N}_{\mathrm{HET} \mid \text { Unb }}$ is the number of helium candidate events activating both the HET and the UNBT, while $\mathrm{N}_{\text {Unb }}$ is the number of Unbiased events. On the left panel of Fig. 3 we present the HET efficiency as a function of the BGO energy deposition computed both for flight and helium MC data. The increasing of the error bars for on-orbit data for higher energies is a consequence of the UNBT pre-scaling. The resulting difference between on-orbit and MC data is found to be within $\sim 4 \%$ and this value has been taken into account as the systematic uncertainty $\sigma_{\text {HET }}$ due to HET affecting our measurement of the helium energy spectrum.

\subsection{Track Reconstruction efficiency}

The efficiency related to the STK track reconstruction has been estimated with the following formula:

$$
\varepsilon_{\text {Track }}=\frac{\mathrm{N}_{\mathrm{PSD}|\mathrm{STK}| \mathrm{BGO}}}{\mathrm{N}_{\mathrm{PSD} \mid \mathrm{BGO}}}
$$

where $\mathrm{N}_{\mathrm{PSD}|\mathrm{STK}| \mathrm{BGO}}$ is the number of helium candidate events selected by our analysis and $\mathrm{N}_{\mathrm{PSD} \mid \mathrm{BGO}}$ is the number of events selected by applying a tracking requirement which only asks for a match between the BGO shower axis and the PSD signals. On the right side of Fig.3, the track reconstruction efficiency is shown both for on-orbit and MC data as a function of different bins of the BGO 

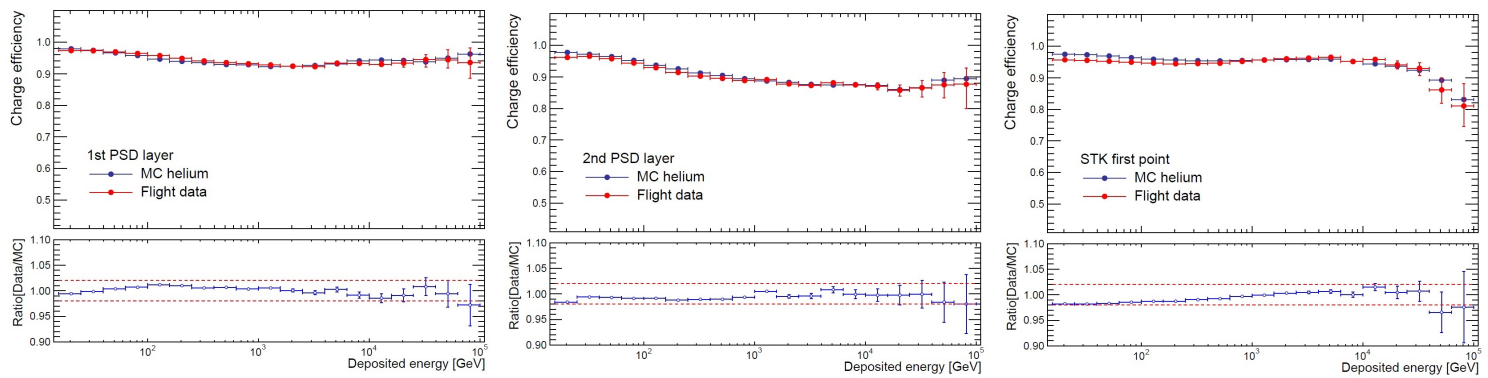

Figure 4: Charge reconstruction efficiencies for the first PSD layer (Left), the second PSD layer (Middle) and the first STK cluster point (Right) as a function of the energy deposited inside the BGO calorimeter. The agreement between flight data (in red) and MC simulations (in blue) is within $\sim 2 \%$ for all the three cases.

energy deposition. On-orbit and MC data are found to be consistent within $\sim 0.5 \%$ and this value has been taken into account as systematic uncertainty due to track reconstruction $\sigma_{\text {Track }}$.

\subsection{Charge Reconstruction efficiency}

An independent evaluation of the charge reconstruction efficiency for the two PSD layers has been carried out by using the charge measurement provided by the first cluster point of the track in the STK. The efficiency for the upper layer of the PSD is hence computed as follows:

$$
\varepsilon_{\text {PSD1st }}=\frac{\mathrm{N}_{\text {PSD1st|PSD2nd|STK1st }}}{\mathrm{N}_{\mathrm{PSD} 2 \mathrm{nd} \mid \mathrm{STK} 1 \mathrm{st}}}
$$

where $\mathrm{N}_{\text {PSD1st|PSD2nd|STK1st }}$ is the number of helium candidate events selected by using the charge measurement provided by the full PSD and the STK, while $\mathrm{N}_{\text {PSD2nd|STK1st }}$ are those events selected by the combined charge measurements of the only 2nd PSD layer with the STK. The same procedure has been adopted for the efficiency related to the second PSD layer. The results are presented in the left and middle panels of Fig.4, where the agreement between on-orbit and MC simulation data is found to be within $\sim 2 \%$ for both the PSD layers.

The charge efficiency for the first STK cluster point is given by:

$$
\varepsilon_{\text {STK1st }}=\frac{\mathrm{N}_{\text {STK1st|PSD }}}{\mathrm{N}_{\mathrm{PSD}}}
$$

by comparing the full event sample with the one selected with the only information provided by the PSD. The right panel of Fig. 4 shows that also in this case the difference between on-orbit and MC simulation data is found to be within $\sim 2 \%$. We take into account all of these contributions for the charge reconstruction efficiency $\sigma_{\text {Charge }}$.

\section{Results and discussion}

The left panel of Fig.5 shows the effective acceptance as a function of the incident energy for the helium candidate event sample resulting from our analysis selection criteria. More in detail, we computed the acceptance at a given $i$-th bin of incident energy by using the following formula:

$$
\mathrm{A}_{\mathrm{eff}, i}=\mathrm{A}_{\mathrm{gen}} \times \frac{\mathrm{N}_{\mathrm{pass}, i}}{\mathrm{~N}_{\mathrm{gen}, i}}
$$

where $\mathrm{A}_{\text {gen }}$ is the geometrical factor used for MC simulations, $\mathrm{N}_{\text {pass }, i}$ is the number of selected events in the $i$-th bin of primary energy, and $\mathrm{N}_{\mathrm{gen}, i}$ is the total number of generated events in the 
same energy bin.

In the right panel of Fig.5 we present the final DAMPE measurement of the $\mathrm{E}^{2.6}$ weighted helium flux as a function of the energy per nucleon, compared with the results provided by previous experiments. This result comes from the adoption of an unfolding procedure [16] which reconstructs the primary energy for the incoming helium nuclei from their measured energy. This procedure has been necessary, because the limited thickness of the DAMPE BGO calorimeter $(\sim 1.62$ interaction lenghts) do not allow the particle to deposit its total energy inside the detector. Furthermore, two corrections have been applied to the energy measurements. The first one consists in a method based on the study of MC simulations and developed in order to correct events for which the saturation of one or more BGO bars occurs [17]. The second correction takes into account the Birk's quenching effect that could occur inside the calorimeter [18] and it is based on the study of dedicated MC simulations in which we reproduced this effect.

The error bars of the DAMPE helium spectrum represent the statistical uncertainties. The inner dashed band instead describes the total systematic uncertainty due to the analysis and given by:

$$
\sigma_{\text {ana }}=\sqrt{\sigma_{\mathrm{HET}}^{2}+\sigma_{\text {Track }}^{2}+\sigma_{\text {Charge }}^{2}+\sigma_{\text {bg }}^{2}+\sigma_{\text {unf }}^{2}+\sigma_{\text {iso }}^{2}} \simeq 5.6 \% .
$$

The uncertainty due to proton background has been estimated to be $\sigma_{\mathrm{bg}} \sim 1 \%-1.5 \%$ by varying the PSD charge selection cut described in Eq. 1 by $5 \%$ and evaluating the difference between the results. The uncertainty due to the unfolding procedure $\sigma_{\mathrm{unf}} \sim 1 \%$ has been evaluated both re-weighting the MC simulations by varying the spectral index from 2.0 to 3.0 and checking the differences with the result obtained by repeating the analysis with $14 \mathrm{BGO}$ layers. The $\sigma_{\text {iso }}$ refers to the uncertainty due to the ${ }^{3} \mathrm{He} /{ }^{4} \mathrm{He}$ isotope ratio affecting the computation of the nucleon average number, which is found to be $\sim 0.2 \%$ for energies lower than $100 \mathrm{GeV}$ and expected to be smaller at higher energies. The uncertainties due to the hadronic interaction models are described by the outer dashed band. In order to estimate it, we applied the same analysis procedure to helium events generated by FLUKA simulations [14] and the difference of the final flux with the one based on GEANT4 simulations is found to be $\sim 12 \%-15 \%$ for primary energies higher than $300 \mathrm{GeV}$. For lower energies, we used instead test beam data and obtained a resulting systematic uncertainty of $\sim 13 \%$ below $300 \mathrm{GeV}$. The helium energy spectrum measured by the DAMPE [7] in the energy range from $70 \mathrm{GeV}$ to $80 \mathrm{TeV}$ can play a key role in the understanding of the Galactic $\mathrm{CR}$ origin, propagation and acceleration. This measurement confirms the observation of a spectral hardening at TeV-energies previously highlighted by other experiments and clearly shows an evidence of a spectral softening at tens of TeV. From the fit of the spectrum with a Smoothly Broken Power-Low (SBPL) function, we obtained an hardening break energy of $1.25_{-0.12}^{+0.15} \mathrm{TeV}$ with a significance of $\sim 24.6 \sigma$, while the softening break energy is $34.4_{-9.8}^{+6.7} \mathrm{TeV}$ with a significance of $\sim 4.3 \sigma$. By comparing the implications of this result with the softening observed by DAMPE in the proton energy spectrum [5], it turns out a charge-dependent softening energy even if a mass-dependence of the structure cannot be ruled out.

\section{References}

[1] M. Anguilar et al. (AMS Collaboration), Phys. Rev. Lett. 115, 211101 (2015); 119, 251101 (2017). 

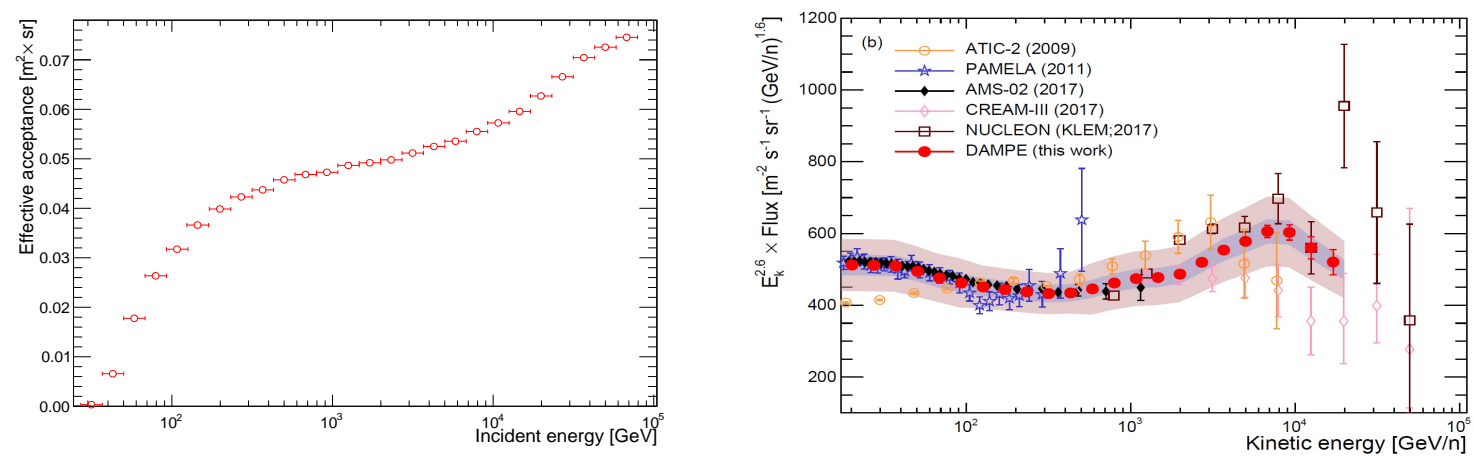

Figure 5: (Left) Effective acceptance for the helium MC events surviving the analysis selection cuts. (Right) Helium flux weighted with $\mathrm{E}^{2.6}$ as a function of the primary energy per nucleon, compared with previous measurements performed by AMS-02 [1], CREAM I+III combined [2], PAMELA [3], ATIC-2 [4] and NUCLEON (KLEM) [6]. The error bars describe the statistical uncertainty, the inner dashed area refers to the systematic uncertainties due to the analysis, while the outer dashed band defines the systematics due to the adopted hadronic model.

[2] H. S. Ahn et al. (CREAM Collaboration), Astrophys. J. Lett. 714, L89 (2010); Y. S. Yoon et al. (CREAM Collaboration), Astrophys. J. 839, 1 (2017).

[3] O. Adriani et al. (PAMELA Collaboration), Science 332, 69 (2011); Adv. Space Res. 51, 219 (2013).

[4] A.D. Panov et al. (ATIC Collaboration), Bull. Russ. Acad. Sci. Phys. 73, 564 (2009).

[5] Q. An et al. (DAMPE Collaboration), Sci. Adv. 5, eaax3793, (2019).

[6] E. Atkin et al., (NUCLEON Collaboration), Cosmol. Astropart. Phys. 07, 020 (2017).

[7] F. Alemanno et al. (DAMPE Collaboration), Phys. Rev. Lett. 126, 201102 (2021).

[8] J. Chang et al. (DAMPE Collaboration), Astropart. Phys. 95, 6 (2017).

[9] Y. H. Yu et al., Astropart. Phys. 94, 1 (2017).

[10] P. Azzarello et al., Nucl. Instrum. Methods Phys. Res., Sect.A 831, 378 (2016).

[11] Z. Zhang et al., Nucl. Instrum. Methods Phys. Res., Sect. A 836, 98 (2016).

[12] Y. Y. Huang et al., Res. Astron. Astrophys. 20, 153 (2020).

[13] S. Agostinelli et al., Nucl. Instrum. Methods Phys. Res., Sect. A 506, 250 (2003).

[14] T. T. Bohlen et al., Nucl. Data Sheets 120, 211 (2014).

[15] E. Thébault et al., Earth Planets Space 67, 79 (2015).

[16] G. D’Agostini, Nucl. Instrum. Meth. A 362, 487 (1995).

[17] C. Yue et al., Nucl. Instrum. Methods Phys. Res., Sect. A 984, 164645 (2020).

[18] J. B. Birks, Proc. Phys. Soc. London Sect. A 64, 874 (1951). 


\section{Full Authors List: DAMPE ${ }^{\dagger}$ Collaboration}

F. Alemanno ${ }^{1,2}$, Q. An ${ }^{3,4}$, P. Azzarello ${ }^{5}$, F. C. T. Barbato ${ }^{1,2}$, P. Bernardini ${ }^{6,7}$, X. J. Bi ${ }^{8,9}$, M. S. Cai ${ }^{10,11}$, E. Casilli6, ${ }^{6,7}$, E. Catanzani ${ }^{12}$, J. Chang ${ }^{10,11}$, D. Y. Chen ${ }^{10}$, J. L. Chen ${ }^{13}$, Z. F. Chen ${ }^{10,11}$, M. Y. Cui ${ }^{10}$, T. S. Cui ${ }^{14}$, Y. X. Cui ${ }^{10,11}$, H. T. Dai ${ }^{3,4}$, A. De Benedittis ${ }^{6,7}$, I. De Mitri ${ }^{1,2}$, F. de Palma ${ }^{6,7}$, M. Deliyergiyev ${ }^{5}$, M. Di Santo ${ }^{1,2}$, Q. Ding ${ }^{10,11}$, T. K. Dong ${ }^{10}$, Z. X. Dong ${ }^{14}$, G. Donvito ${ }^{15}$, D. Droz ${ }^{5}$, J.

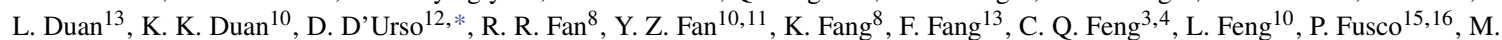

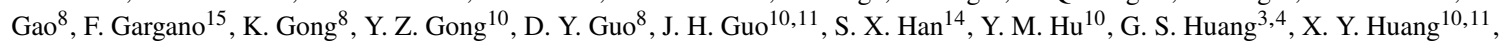

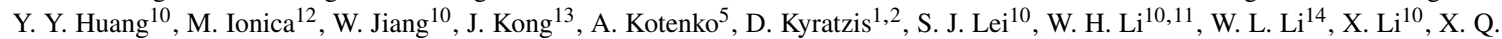

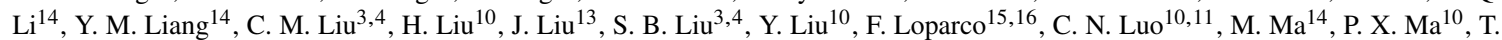

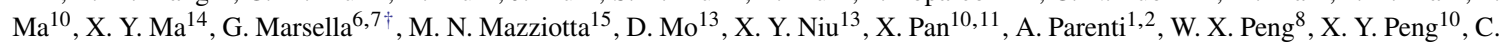
Perrina $^{5,+}$, R. Qiao ${ }^{8}$, J. N. Rao ${ }^{14}$, A. Ruina ${ }^{5}$, M. M. Salinas ${ }^{5}$, G. Z. Shang ${ }^{14}$, W. H. Shen ${ }^{14}$, Z. Q. Shen ${ }^{10}$, Z. T. Shen ${ }^{3,4}$, L. Silveri ${ }^{1,2}$, J. X. Song ${ }^{14}$, M. Stolpovskiy ${ }^{5}$, H. Su ${ }^{13}$, M. Su${ }^{17}$, H. R. Sun ${ }^{3,4}$, Z. Y. Sun ${ }^{13}$, A. Surdo ${ }^{7}$, X. J. Teng ${ }^{14}$, A. Tykhonov ${ }^{5}$, H. Wang ${ }^{14}$, J. Z.

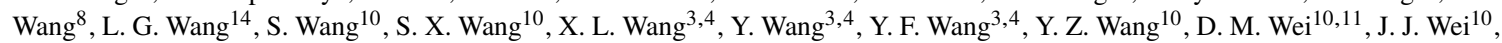
Y. F. Wei ${ }^{3,4}$, D. $\mathrm{Wu}^{8}$, J. Wu ${ }^{10,11}$, L. B. $\mathrm{Wu}^{1,2}$, S. S. $\mathrm{Wu}^{14}$, X. Wu ${ }^{5}$, Z. Q. Xia ${ }^{10}$, H. T. Xu ${ }^{14}$, Z. H. Xu ${ }^{10,11}$, Z. L. Xu ${ }^{10}$, E. H. Xu ${ }^{3,4}$, Z. Z. Xu ${ }^{3,4}$, G. F. Xue ${ }^{14}$, H. B. Yang ${ }^{13}$, P. Yang ${ }^{13}$, Y. Q. Yang ${ }^{13}$, H. J. Yao ${ }^{13}$, Y. H. Yu ${ }^{13}$, G. W. Yuan ${ }^{10,11}$, Q. Yuan ${ }^{10,11}$, C. Yue ${ }^{10}$, J. J. Zang $^{10}$ S. X. Zhang ${ }^{13}$, W. Z. Zhang ${ }^{14}$, Yan Zhang ${ }^{10}$, Yi Zhang ${ }^{10,11}$, Y. J. Zhang ${ }^{13}$, Y. L. Zhang ${ }^{3,4}$, Y. P. Zhang ${ }^{13}$, Y. Q. Zhang ${ }^{10}$, Z. Zhang ${ }^{10}$, Z. Y. Zhang ${ }^{3,4}$, C. Zhao ${ }^{3,4}$, H. Y. Zhao ${ }^{13}$, X. F. Zhao ${ }^{14}$, C. Y. Zhou ${ }^{14}$, and Y. Zhu ${ }^{14}$

${ }^{1}$ Gran Sasso Science Institute (GSSI), Via Iacobucci 2, I-67100 L'Aquila, Italy

${ }^{2}$ Istituto Nazionale di Fisica Nucleare (INFN) -Laboratori Nazionali del Gran Sasso, I-67100 Assergi, L’Aquila, Italy

${ }^{3}$ State Key Laboratory of Particle Detection and Electronics, University of Science and Technology of China, Hefei 230026, China

${ }^{4}$ Department of Modern Physics, University of Science and Technology of China, Hefei 230026, China

${ }^{5}$ Department of Nuclear and Particle Physics, University of Geneva, CH-1211, Switzerland

${ }^{6}$ Dipartimento di Matematica e Fisica E. De Giorgi, Universita“ del Salento, I-73100, Lecce, Italy

${ }^{7}$ Istituto Nazionale di Fisica Nucleare (INFN) - Sezione di Lecce, I-73100, Lecce, Italy

${ }^{8}$ Institute of High Energy Physics, Chinese Academy of Sciences, Yuquan Road 19B, Beijing 100049, China

${ }^{9}$ University of Chinese Academy of Sciences, Yuquan Road 19A, Beijing 100049, China

${ }^{10}$ Key Laboratory of Dark Matter and Space Astronomy, Purple Mountain Observatory, Chinese Academy of Sciences, Nanjing 210023 ,

China

${ }^{11}$ School of Astronomy and Space Science, University of Science and Technology of China, Hefei 230026, China

${ }^{12}$ Istituto Nazionale di Fisica Nucleare (INFN) - Sezione di Perugia, I-06123 Perugia, Italy

${ }^{13}$ Institute of Modern Physics, Chinese Academy of Sciences, Nanchang Road 509, Lanzhou 730000, China

${ }^{14}$ National Space Science Center, Chinese Academy of Sciences, Nanertiao 1, Zhongguancun, Haidian district, Beijing 100190, China

${ }^{15}$ Istituto Nazionale di Fisica Nucleare (INFN) - Sezione di Bari, I-70125, Bari, Italy

${ }^{16}$ Dipartimento di Fisica "M. Merlin" dell'Universita' e del Politecnico di Bari, I-70126, Bari, Italy

${ }^{17}$ Department of Physics and Laboratory for Space Research, the University of Hong Kong, Pok Fu Lam, Hong Kong SAR, China

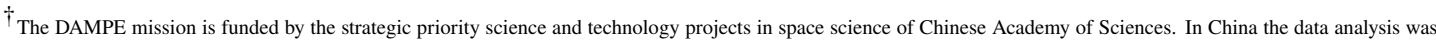
supported in part by the National Key Research and Development Program of China (No. 2016YFA0400200), the National Natural Science Foundation of China (Nos. 11525313, 11622327, 11722328, U1738133, U1738205, U1738207, U1738208), the strategic priority science and technology projects of Chinese Academy of Sciences (No. XDA15051100), the 100 Talents Program of Chinese Academy of Sciences, and the Young Elite Scientists Sponsorship Program. In Europe the activities and the data analysis are supported by the Swiss National Science Foundation (SNSF), Switzerland; the National Institute for Nuclear Physics (INFN), Italy.

*Now at Dipartimento di Chimica e Farmacia, Universita“ di Sassari, I-07100, Sassari, Italy.

$\dagger$ Now at Dipartimento di Fisica e Chimica "E. Segre“", Universita“ degli Studi di Palermo, via delle Scienze ed. 17, I-90128 Palermo, Italy.

¥Also at Institute of Physics, Ecole Polytechnique Federale de Lausanne (EPFL), CH-1015 Lausanne, Switzerland.

$\S$ Also at School of Physics and Electronic Engineering, Linyi University, Linyi 276000, China.
} 